\title{
ASYNCHRONOUS EXCITERS AND STABILIZERS OF ARC. ANALYSIS AND CALCULATION PROCEDURE. Part 2
}

\author{
N.M. MAKHLIN ${ }^{1}$ and A.E. KOROTYNSKY ${ }^{2}$ \\ ${ }^{1}$ State Enterprise «Researh Engineering Center of Welding and Control «Power Engineering» \\ of E.O. Paton Electric Welding Institute, NASU \\ 11 Bozhenko Str., 03680, Kiev, Ukraine. E-mail: electro@paton.kiev.ua \\ ${ }^{2}$ E.O. Paton Electric Welding Institute, NASU \\ 11 Bozhenko Str., 03680, Kiev, Ukraine. E-mail: office@paton.kiev.ua
}

\begin{abstract}
Issues of analysis of processes in electronic voltage boosters of asynchronous type with series circuit of main or pilot arcs are considered. They have configuration of forming circuit, in which capacitive storage and inductance of primary winding of pulse step-up transformer have series connection, and switching key of the circuit is parallel. Part 2 of the work provides for the results of analysis of circuits of increased voltage pulse generators, forming asynchronous exciters and stabilizers of arcing. Description is given to circuit solutions and issues of analysis of charge-discharge circuit of asynchronous exciters and stabilizers of arcing, built based on method of resonant pumping, are reflected. Analysis of processes in the circuit of asynchronous exciters and stabilizers of arcing is carried out using solutions of the equations known in theoretical electrical engineering. Engineering procedure is proposed based on these solutions for calculation of main components in functional assemblies of asynchronous exciters and stabilizers of arcing and recommendations of selection of their element base are given. 29 Ref., 2 Tables, 6 Figures.
\end{abstract}

Keywords: arc and plasma welding, asynchronous exciters and stabilizers of arc, generator of increased voltage pulses, pulse transformer, cores, ferrites, sprayed iron, device for input of high-voltage pulses, resonant pumping

Efficiency of contact-free methods for initiation of stationary arc discharge by ionizing of interelectrode gap due to injection of pulses of high or increased voltage in it mainly depends on method of input of these pulses in arc circuit and parameters of high-voltage pulses, generated by special voltage booster-exciters and stabilizers of arc. Series of works, for example [1-5], are dedicated to structural design of such electronic devices and their circuit design. However, issues of analysis of electromagnetic processes in functional assemblies of such devices and procedures of their calculation are considered in insufficient for practice volume. Work [6] bridges this gap to some extent. It is dedicated to circuit design, analysis and procedure for calculation of electronic voltage boosters with independent alternating voltage supply (mainly of industrial frequency) with series circuit of main or pilot arc. Work [7] studies the issues of circuit design, analysis and procedure for calculation of charging devices (CD) of asynchronous exciters and stabilizers of arc (AESA), made based on key scheme with dosing reactor or voltage multiplier (VM) designed by Cockcroft-Walton scheme. Moreover, similar issues concerning such important functional assembly of AESA as generator of increased voltage pulses (GVP), which is imbedded

(c) N.M. MAKHLIN and A.E. KOROTYNSKY, 2015 in AESA regardless the method of realizing or CD scheme, have fragment representation in known references up to the present time. This promotes for some difficulties in development and designing of AESA. Significant difficulties appear in development and designing of AESA, based on application resonant pumping method for charge of GVP capacitive storage, due to absence of woks providing the results of analysis of processes in circuits of similar devices and procedure for calculation of their assemblies and units.

Aim of the present work lies in analysis of processes in GVP, imbedded in AESA, as well as in functional assemblies of AESA, based on method of resonant pumping, using for analysis the methods of investigation of linear circuits known in theoretical electrical engineering, making recommendations on calculation and selection of components of devices of this group following the results of their analysis and experience of development, manufacture and practical application.

Peculiarities of analysis and calculation of GVP. Principle of AESA operation is based on periodic charging of GVP capacitive storage and its further discharge via primary winding of pulse step-up transformer, which secondary winding injectes pulses of high or increased voltage in the circuit of main or pilot arc [1, 2, 7].

Figure 1, $a$ shows a structural-functional scheme of AESA with CD based on key scheme with dosing reactor or CD based on VM and series circuit of direct or alternating current arc. Basic variant of GVP of such AESA represents 
itself series $L C$-ringing circuit including capacitive storage $C_{\mathrm{c}}$, inductance $L_{\mathrm{c}}$ (inductance of primary winding $I$ of pulse step-up transformer $T V 1)$ and semiconductor switching element $\mathrm{K}$ (key) with control circuit 1.

Formation of increased voltage pulses in GVP circuit is carried out due to transition process taking place at periodic turn-on of semiconductor key K. Calculation equivalent scheme, shown in Figure 2, $a$, can be used for analysis of transition process in GVP circuit. At that the following assumptions, which virtually have no effect on analysis quality, are reasonable to take into account:

1) $R_{\mathrm{k}}=R_{\mathrm{k} . \mathrm{d}}+E S R_{C}+R_{L}+R_{\text {c.w }}$, where $\mathrm{R}_{\mathrm{k} . \mathrm{d}}$ is the dynamic resistance of turn-on key $\mathrm{K} ; E R S_{C}$ is the equivalent series active resistance of GVP capacitive storage; $R_{L}$ is the active resistance of primary winding of pulse step-up transformer $T V 1 ; R_{\text {c.w }}$ is the active resistance of GVP connecting wires;

2) inductance $L_{\mathrm{c}}$ of primary winding $I$ of pulse step-up transformer $T V 1$ is linear, equal to initial one and does not depend on current passing in it;

3) $\tau_{\text {trans }}<<T_{i}$, where $\tau_{\text {trans }}$ is the duration of transition process; $T_{i}$ is the period of turn-on frequency of semiconductor key $\mathrm{K}$;

4) $R_{\mathrm{c}} / 2 L_{\mathrm{c}}<1 / \sqrt{L_{\mathrm{c}} C_{\mathrm{c}}}$

5) $U_{\text {out }}=U_{L} W_{2} / W_{1}$, where $U_{\text {out }}$ is the output voltage of AESA (voltage on secondary winding of pulse step-up transformer $T V 1$ ); $U_{L}$ is the voltage on $L_{\mathrm{c}}$ inductance; $W_{1}$ and $W_{2}$ are the number of turns of primary and secondary winding of pulse step-up transformer $T V 1$, respectively.

Transition process under non-zero initial conditions in $R_{\mathrm{c}} C_{\mathrm{c}} L_{\mathrm{c}}$-circuit with preliminary charged capacity for $u_{C}$ voltage on GVP capacitive storage considering made assumptions is described by known differential equation of the second order $[8,9]$

$$
L C \frac{d^{2} u_{C}}{d t^{2}}+R C \frac{d u_{C}}{d t}+u_{C}=U_{C 0},
$$

where $U_{C 0}=U_{C y}$ is the steady-state capacitive storage voltage (at $t \rightarrow \infty, d u_{C} / d t=0$ and $i_{C}(t)=$ $=0$ ), i.e. capacitive storage voltage $C_{\mathrm{c}}$ directly before semiconductor key $\mathrm{K}$ turn-on.

Solutions of equation (1) can be represented in form of expressions by entering designations $R / 2 L=\delta, 1 / L C=\beta_{0}^{2} \beta=\sqrt{\beta_{0}^{2}-\delta^{2}}$ and considering made assumption $\beta_{0}^{2}>\delta^{2}$ :

$$
\begin{gathered}
i_{C}=-\frac{U_{C 0}}{\beta L} \sin \beta t e^{-\beta t}, \\
u_{C}=U_{C 0} \frac{\beta_{0}}{\beta} \cos (\beta t-\gamma) e^{-\delta t},
\end{gathered}
$$

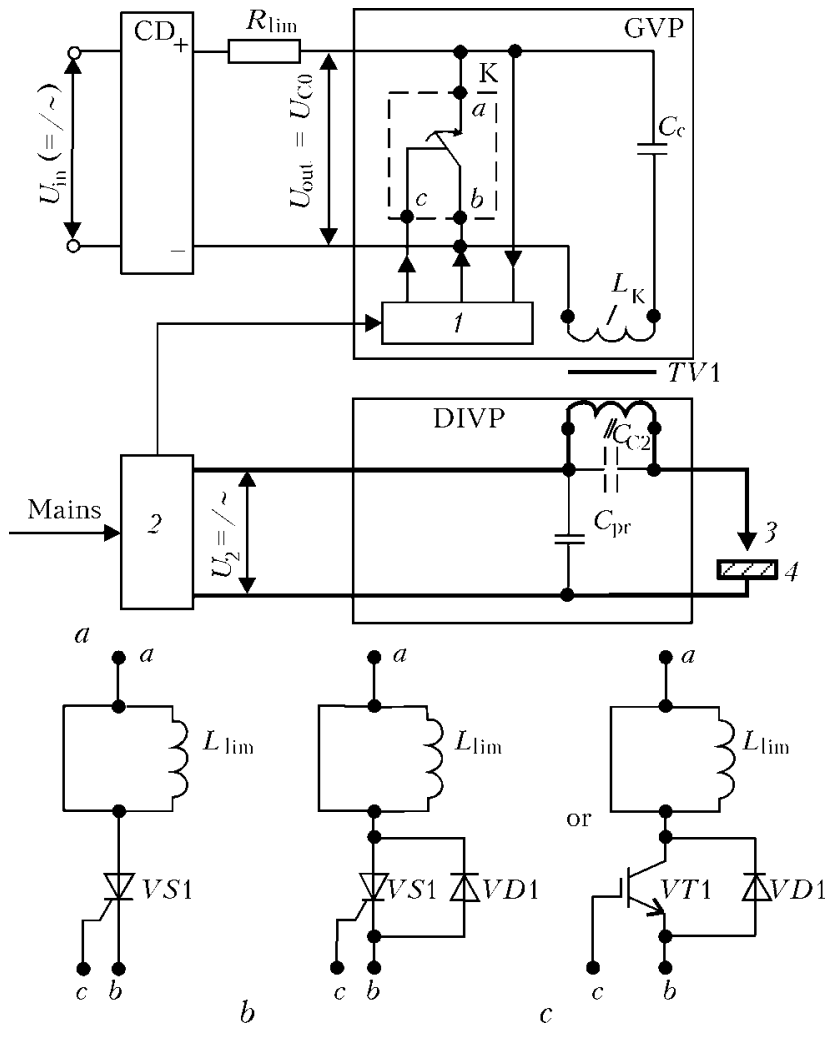

Figure 1. Structural scheme of AESA with series connection to welding circuit $(a)$; electric schematic diagram of semiconductor GVP switching key with unidirectional conduction (b); variants of electrical schematic diagram of semiconductor GVP switching key with two-directional conductivity $(c)$ : 1 - AESA control scheme; 2 - arc power supply; 3 - welding electrode; 4 - part being welded; CD - step-up charging device; $R_{\lim }-$ current-limiting resistor; GVP - generator of increased voltage pulses; $\mathrm{K}-$ semi-conductor switching key of GVP; $C_{c}-$ capacitor of GVP forming circuit; $T V 1$ - step-up pulse transformer with primary $I$ and secondary $I I$ windings; $L_{\mathrm{c}}$ - inductance of GVP forming circuit (inductance of winding $I$ of $T V 1$ transformer); $L_{\lim }$ - additional inductance limiting speed of current buildup through GVP switching key; DIVP device for input of high-voltage pulses to arc circuit; $C_{\mathrm{C} 2}-$ capacitor of additional forming circuit of DIVP; $C_{\mathrm{pr}}-$ protective (block) DIVP capacitor; VS1 - thyristor of semi-conductor GVP switching key; VT1 - transistor of semi-conductor GVP switching key; VD1 - back diode of semi-conductor GVP switching key

where $\operatorname{tg} \gamma=\delta / \beta_{0}$, and «minus» sign for $i_{C}$ indicate direction of discharge current of capacitive storage $C_{c}$ opposite to direction of current of its charge.

Based on relationship $u_{L}=L \frac{d t}{d t}$ and expression (2) inductance transition voltage $L_{\mathrm{c}}$ can be determined by formula

$$
u_{L}=-\frac{U_{C 0}}{\beta}(\beta \cos \beta t-\delta \sin \beta t) e^{-\delta t} .
$$

It can be supposed under made assumptions that $\beta_{0} / \beta \approx 1$ for GVP circuit, and, therefore, formula (3) with accuracy, sufficient for practice, can be represented as

$$
u_{L}=-U_{C 0} \cos (\beta t-\gamma) e^{-\delta t} .
$$



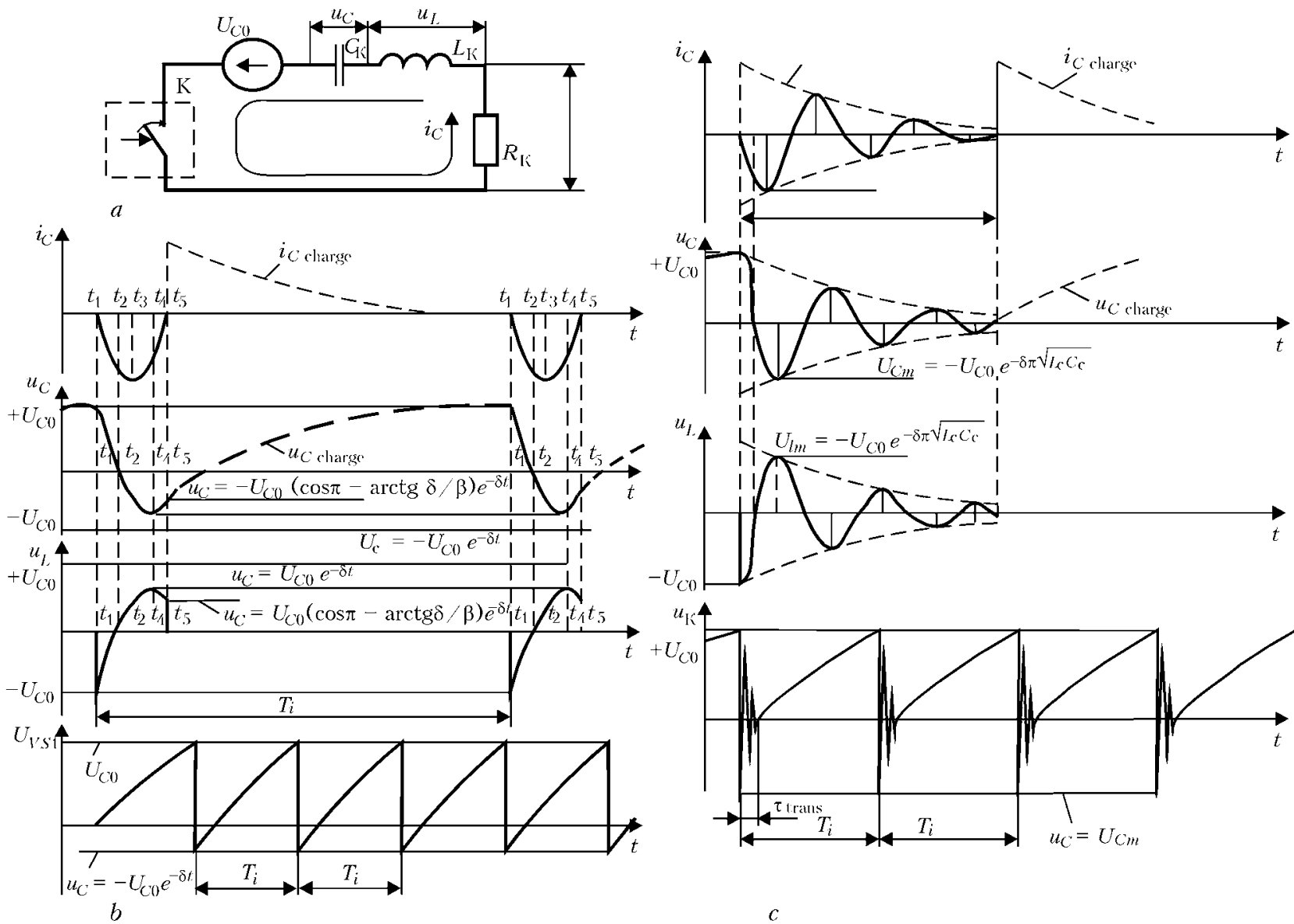

Figure 2. GVP equivalent circuit ( $a$ ), and diagrams of currents and voltages in GVP circuits at unidirectional (b) and two-directional $(c)$ conductance of semi-conductor switching key $\mathrm{K}$

In (2)-(4) $\beta$ represents itself angular frequency of own oscillations at transition process in $R_{\mathrm{c}} C_{\mathrm{c}} L_{\mathrm{c}}$-circuit; $\delta$ is the coefficient of attenuation of these oscillations.

Diagrams of current $i_{C}$, voltages $u_{C}$ and $u_{L}$, reflecting the nature of transition process in $R_{\mathrm{c}} C_{\mathrm{c}} L_{\mathrm{c}}$-circuit of GVP depending on type of conductance (unidirectional or two-directional - see Figure 1, $b$ and $c$, respectively) of key $\mathrm{K}$ are given in Figures 2, $b$ and $c$, respectively.

Process of recharging of capacitive storage $C_{\mathrm{c}}$ via inductance $L_{\mathrm{c}}$ of GVP circuit takes place from the moment of its turn-on $t_{1}$ (Figure $2, b$ ) at unidirectional conductance of key K (thyristor $V S 1$ is usually used as the latter - see Figure 1, b). At that, GVP circuit starts to pass current $i_{C}$, which will be changes by sine law from zero in moment $t_{1}$ to amplitude value $I_{C m}=U_{C 0} / \beta L$ iv $\mu$ o $\mu \varepsilon v \tau t_{3}$, and from amplitude value to zero in moment $t_{5}$. As a consequence, key $\mathrm{K}$ in moment $t_{5}$ will naturally transfer into non-conducting state and this is the moment of beginning of process of GVP capacitive storage charging. Voltage $u_{C}$ of capacitive storage $C_{\mathrm{c}}$ from moment $t_{1}$ starts changing by cosine law from value $+U_{C 0}$ to zero in moment $t_{2}$, and then from amplitude value $U_{C m} \approx-U_{C 0} e^{-\delta t}$ in moment $t_{4}$, and from amplitude value to value $u_{C}=-U_{C 0} \cos (\pi-\operatorname{arctg} \delta / \beta) e^{-\delta t}$ in moment $t_{5}$, from which growing by exponential law takes place providing charging of GVP storage up to $U_{C 0}$ value. In turn, $u_{L}=-U_{C 0}$ voltage appear in moment $t_{1}$ on $L_{\mathrm{c}}$ inductance of GVP circuit, which starts changing by cosine law from $-U_{C 0}$ value to zero in moment $t_{2}$, then from zero to amplitude value $U_{L m} \approx U_{C 0} e^{-\delta t}$ in moment $t_{4}$, and from amplitude value to $u_{L}=U_{C 0} \cos (\pi-$ $-\operatorname{arctg} \delta / \beta) e^{-\delta t}$ value in moment $t_{5}$, after what it immediately drops to zero. Time shifts $\Delta t=t_{3}-$ $-t_{2}=t_{5}-t_{4}$ depend on $Q$-factor of GVP $R_{\mathrm{c}} C_{\mathrm{c}} L_{\mathrm{c}}-$ circuit, which is determined by expression

$$
Q=\frac{\sqrt{L_{\mathrm{c}} C_{\mathrm{c}}}}{R_{\mathrm{c}}} .
$$

Duration of transition process at unidirectional conduction of key $\mathrm{K}$ can be determined by formula

$$
\tau_{\text {trans }}=\pi \sqrt{L_{\mathrm{c}} C_{\mathrm{c}}} .
$$

Two-directional conductance of semi-conductor key K (see Figure 1,c) from moment of its connection to GVP circuit will provoke classical transition process with attenuating oscillations, described by known equation (1), solution of which are expressions (2)-(4). Figure 2, $c$ shows curves of change of current $i_{C}$ and voltages $u_{C}$ and $u_{L}$ during transition process with attenuating 
oscillations. It is known from $[8,9]$ that phase shifts between $i_{C}$ current and $u_{C}$ and $u_{L}$ voltages and duration of transition process at two-directional conductance of key $\mathrm{K}$ depend on GVP $R_{\mathrm{c}} C_{\mathrm{c}} L_{\mathrm{c}}$-circuit $Q$-factor, at that duration of transition process with attenuating oscillations is determined by relationship

$$
\tau_{\text {trans }}=\frac{6 L_{\mathrm{c}}}{R_{\mathrm{c}}} .
$$

Process of charging of capacitive storage $C_{\mathrm{c}}$ to $U_{C 0}$ value starts repeating from moment of ending of transition process attenuating oscillations in GVP circuit. One of the peculiarities of transition process with attenuating oscillations is virtual symmetry of curves of current $i_{C}$ and voltages $u_{C}$ and $u_{L}$ relatively to zero, that provides for the possibility of efficient use of pulse step-up transformer $T V 1$ (see Figure 1, a). At that, such a transformer can operate at maximum allowable span of induction in its core, i.e. from $+B_{\text {sat }}$ to $-B_{\text {sat }}$.

Another peculiarity of GVP circuit, key K of which has two-directional conductance, lies in the fact that duration of transition process with attenuating oscillation exceeds duration of transition process at unidirectional conductance of key $\mathrm{K}$. This promotes for more continuous retention of plasma in impact ionization state in interelectrode gap. This is particularly important in the cases, when AESA is used for excitation of low-amperage arcs, as well as stabilizing of burning process of low-stable alternating current arcs (for example TIG welding of aluminum and its alloys or MMA welding by electrodes with lime fluorspar coating). The latter are characterized by quick deionization of inter electrode gap at change of arc current polarity [4, 10].

However, it should be noted that in comparison with GVP, which consists of key with unidirectional conductance, realizing of two-directional conductance of key $\mathrm{K}$ requires some complication and application of semiconductor devices with higher allowable back voltages as well as key control scheme capable to provide duration of control signals being neither less, not more than duration of transition process with attenuating oscillations.

Nonfailure operation of semiconductor key K requires inductance value $L_{\mathrm{c}}$ of GVP circuit which provides for fulfillment of the requirements $I_{\mathrm{Cm}}<I_{\mathrm{OS} \text {.OVL max }}$ and $(d i / d t)_{\mathrm{c}}<$ $<(d i / d t)_{\mathrm{cr}}$, where $I_{\mathrm{OS} . \mathrm{OVL} \max }$ is the maximum allowable repeatable overload current of on-state semiconductor key (maximum allowable current of operating overload $) ;(d i / d t)_{\mathrm{c}}$ is the maximum value of speed of current buildup $i_{C}$ in GVP circuit; $(d i / d t)_{\text {cr }}$ is the value of critical speed of current buildup at on-state key K. Maximum amplitude value of $I_{\mathrm{C} m}$ current in GVP circuit according to (2) can be determined by expression

$$
I_{\mathrm{C} m}=U_{C 0} \sqrt{\frac{C_{\mathrm{c}}}{L_{\mathrm{c}}}},
$$

and in real models of AESA makes form 80 to 445 A. Taking into account that duration of such current does not exceed few microseconds and maximum speed of current buildup $i_{C}=50-$ $80 \mathrm{~A} / \mathrm{s}$, fulfillment of the requirement on nonfailure operation of on-state key $\mathrm{K}$ based on allowable current of operating overload $I_{\text {OS.OVL max }}$ and critical speed of current buildup in AESA is totally reachable [11].

Moreover, calculation and designing of pulse step-up transformer (PT) of AESA promote some difficulties due to high values of magnetic field intensity $H$ in the core, caused by amplitude values of current $I_{\mathrm{C} m}$ in GVP circuit. It is known fact that inductance in PT core at its change from $-B_{\text {sat }}$ to $+B_{\text {sat }}$ is related with intensity $H$ by $B=$ $=\mu H$ relationship, where $\mu=\mu_{0} \mu_{\mathrm{e}}$ is the magnetic permeability of PT core material; $\mu_{0}$ is the magnetic constant equal $4 \pi \cdot 10^{-7} ; \mu_{\mathrm{e}}$ is the effective magnetic permeability of the core (for cores without gap $\mu_{\mathrm{e}}$ value can be taken equal initial magnetic permeability $\left.\mu_{\text {init }}\right)$. Intensity $H$ in PT core is determined as [12]

$$
H=\frac{I_{\mathrm{C} m} W_{1}}{l_{\mathrm{c}}},
$$

where $W_{1}$ is the number of turns of PT primary winding; $l_{\mathrm{c}}$ is the length of average line of magnetic inductance in the core. Table 1 gives the largest calculation values of $H_{\max }$ and $B_{\max }$ depending on calculated according to (8) $I_{\mathrm{Cm}}$ current values at $U_{C 0}=700 \mathrm{~V}, l_{\mathrm{c}}=0.44 \mathrm{~m}, W_{1}=1, \mu_{\mathrm{e}}=1.58 \cdot 10^{3}$ and typical for GVP values of $C_{\mathrm{c}}$ and $L_{\mathrm{c}}$.

It follows form data of Table 1 that application of single ferrite cores without gap with acceptable geometry parameters, in which value of saturation induction $B_{\text {sat }}$ can make $0.48-0.50$ T [13], at typical for GVP values of $I_{\mathrm{C} m}, C_{\mathrm{c}}, L_{\mathrm{c}}$ and $\mu_{\mathrm{init}}$ is virtually impossible in PT of AESA.

Application of such widespread method of increase of $H_{\max }$ allowable value as introduction of gap with length not higher than $1 \%$ of $l_{\mathrm{c}}$ in the core [12], at which induction in PT ferrite core does not exceed $B_{\text {sat }}$ value, is more effective. In this case $\mu_{\mathrm{e}}$ value can make $(0.12-0.14) \mu_{\text {init }}$ (due to which $H_{\max }$ value can reach $2700-$ $3000 \mathrm{~A} / \mathrm{m}$ depending on $B_{\text {sat }}$ ). However, reduction of $\mu_{\text {init }}$ value provokes for proportional decrease of value of induction coefficient $A_{L}=$ $=L_{\mathrm{c}} / W_{1}^{2}=\mu_{0} \mu_{\mathrm{e}} A_{\mathrm{e}} / l_{\mathrm{c}}$, where $A_{\mathrm{e}}$ is the effective area of section of single core. This provides for the necessity of application of PT core consisting 
SCIENTIFIC AND TECHNICAL

Table 1. Dependence of $B_{\max }$ on $H_{\max }$ at different values of $C_{\mathrm{c}} / L_{\mathrm{c}}$ relationship

\begin{tabular}{|c|c|c|c|c|c|c|}
\hline \multirow{2}{*}{$C_{\mathrm{c}}, \mu \mathrm{F}$} & \multicolumn{2}{|c|}{$L_{\mathrm{c}}=20 \mu \mathrm{H}$} & \multicolumn{2}{|c|}{$L_{\mathrm{c}}=14 \mu \mathrm{H}$} & \multicolumn{2}{|c|}{$L_{\mathrm{c}}=1 \mu \mathrm{H}$} \\
\hline & $H_{\max }, \mathrm{A} / \mathrm{m}$ & $B_{\text {max }}, \mathrm{T}$ & $H_{\max }, \mathrm{A} / \mathrm{m}$ & $B_{\max }, \mathrm{T}$ & $H_{\max }, \mathrm{A} / \mathrm{m}$ & $B_{\text {max }}, \mathrm{T}$ \\
\hline 0.25 & 178 & 0.353 & 213 & 0.423 & 252 & 0.499 \\
\hline 0.50 & 252 & 0.499 & 301 & 0.597 & 356 & 0.706 \\
\hline 1 & 356 & 0.706 & 424 & 0.841 & 503 & 0.999 \\
\hline 2 & 503 & 0.999 & 602 & 1.194 & 712 & 1.412 \\
\hline 3 & 616 & 1.223 & 736 & 1.461 & 871 & 1.729 \\
\hline 4 & 712 & 1.412 & 851 & 1.689 & 1006 & 1.997 \\
\hline
\end{tabular}

from several single cores, in order to receive acceptable value of $L_{c}$. Besides, introduction of gap does not eliminate the necessity of application of ferrite cores with significant length of median line of magnetic induction $l_{\mathrm{c}}(>0.2 \mathrm{~m})$, that inevitably provokes for increase of weight, dimension size and price of PT.

Significantly better results can be received at PT designing in form of transformer with completely open rod-type ferrite core (Figure 3), for which expressions [12] are true:

$$
\mu_{\mathrm{e}}=\frac{\mu_{\text {init }}}{1+g\left(\mu_{\text {init }}-1\right)}, \quad g=0.84\left(D / l_{\mathrm{f}}\right)^{1.7},
$$

where $\mu_{\text {init }}$ is the initial magnetic permeability of core; $g$ is the demagnetizing factor of rod-type ferrite core; $D$ is the diameter; and $l_{\mathrm{f}}$ is the length of ferrite rod. Such PT structure provides for significant reduction of its weight-dimension indices and virtually eliminated possibility of its core saturation, that is achieved due to low $\mu_{e}$ typical for this structure. Moreover, this predetermines low value of inductance $L_{W 1}$ of PT primary winding (not more than $7 \mu \mathrm{H}$ ), which can be provided only at increased number of turns of primary winding (usually up to 11-12) and, respectively, secondary winding of PT. In addition to this, low value of inductance $L_{W 1}$ provokes necessity of introduction of additional inductance $L_{\text {add }}$ (see Figure $1, b, c$ ), having series connection to switching key $\mathrm{K}$, in GVP scheme for providing conditions of nonfailure operation of key K. Fulfillment of $L_{\mathrm{c}}=L_{W 1}+L_{\text {add }}>$ $>U_{C 0} /(d i / d t)_{\text {cr }}$ requirement is one of them.

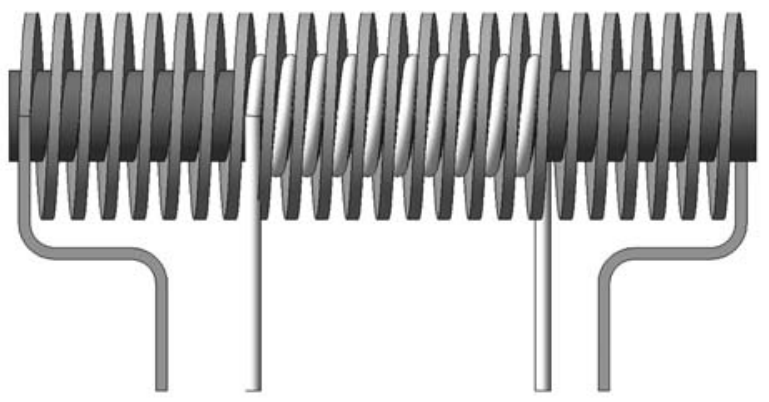

Figure 3. Structure of AESA step-up pulse transformer with completely open rod-type ferrite core
Production of secondary winding in form of single-layer winding, having edgewise winding due to necessity of distribution of this winding along the whole length of the core and providing minimum possible gap between winding and core, is also typical for PT with rod-type ferrite core. It should be noted that up to now literature does not include information on dependence and procedure of calculation of demagnetizing factor for open ferromagnetic cores allowing analytically strict determination of their effective magnetic permeability. This is why experimental data are used as a rule. Besides, number of turns of secondary winding in such PT is limited by length of industrially produced ferrite rods and usually does not exceed 28-30. Nevertheless, PT with rod-type ferrite core finds more and more application in AESA. It is indicated by practice of design and manufacture of welding inverters (mainly for TIG welding) by leading companies from Sweden, Italy, Finland, Ukraine, China, Russia and other countries.

Analysis of possible values of amplitude and frequency GVP parameters allows making a conclusion that core of pulse step-up GVP transformer shall be made from material giving low effective magnetic permeability $\mu_{\mathrm{e}}$ and, simultaneously, sufficiently high saturation induction $B_{\text {sat }}$ for receiving optimized electromagnetic and weight-dimension characteristics. Such properties are characteristic for cores from iron powder (IPC), being widely used in the recent years in inductance elements of switching power supplies and representing itself ferromagnetic structures with distributed air gap [14].

High saturation inductance typical for iron $\left(B_{\text {sat }} \leq 1.5 \mathrm{~T}\right)$ in combination with distributed gap provides for the possibility of producing a core with low effective magnetic permeability $\left(\mu_{\mathrm{e}} \leq 120\right)$ and allowable values $H_{\max }=8000-$ $20000 \mathrm{~A} / \mathrm{m}$, capable to function in wide range of frequencies (more $100 \mathrm{kHz}$ ) [15-17]. Significant advantage of IPC is the fact that iron powder has the lowest price among the most widespread materials with high saturation inductance. Such 
IPC characteristics provides for the possibility to create compact PT with rational number of winding turns and optimized weight-dimension indices. IPC application allows reaching inductance values of primary winding around $8 \mu \mathrm{H}$ and more in GVP pulse step-up transformer. At that, calculation of PT with IPC can be made using known and, to significant extent, processed procedures $[12,17]$ as well as results of additional experimental investigations. It can be expected that PT with IPC as well as chokes with such cores receive wide distribution in the future years not only in AESA, but welding inverters and converters.

It is noted in [6] that resistance of inter electrode gap is sufficiently high (from several tens to hundred of $\mathrm{kOhms}$ ) in breakdown and spark discharge being referred to independent unstable discharges in gases. Therefore, effect of welding circuit, including DIVP circuits, on GVP parameters is negligibly small and expressions (2)(8) are true for open-circuit AESA mode as well as spark discharge mode (up to the moment of transition of spark discharge of interelectrode gap to stationary arc).

Expressions (2)-(10) and results of experimental investigations as well as experience of designing and operation of AESA can be used for calculation of parameters and selection of components of basic GVP variant (see Figure 1, $a$ ) in the following way:

1. Calculation and nominal value of capacity $C_{\mathrm{c}}$ of GVP storage and type of applied capacitors are determined based on necessary value of energy of pulses being injected in the circuit of main and pilot arc in accordance with recommendations given in [6, 7] and according to relationship $C_{\mathrm{c}} \geq 2 W_{C} / U_{C 0 \text { min }}^{2}$, where $W_{C}$ is the energy accumulated in GVP capacitive storage at its charge; $U_{C 0 \text { min }}$ is the minimum possible value of steady-state charge voltage of capacitive storage in specific CD scheme. At that, the largest allowable voltage of direct current $U_{V D C}$ for capacitor (capacitors) of GVP capacitive storage shall fulfill the condition

$$
U_{V D C} \geq 1.6 U_{C 0 \max },
$$

where $U_{C 0 \text { max }}$ is the maximum possible value of steady-state charge voltage of capacitive storage in specific CD scheme.

2. Reference data, indicated, for example in $[11,18-21]$ as well as recommendations of work [6], are used for determination of values of constituents of GVP circuit active resistance, in accordance with which $R_{\mathrm{c}}$ resistance value does not exceed $30 \mathrm{mOhm}$ in most cases.
3. Calculation of inductance of GVP circuit is carried out depending on chosen PT structure, material and geometry of its core.

3.1. If PT design with $\Pi$ - or E-shaped ferrite cores are selected by design or economy issues, than it is reasonable to set values of inductance $L_{\mathrm{c}}$ of primary winding and number of its turns $W_{1}$ for PT calculation as well as determine number of turns $W_{2}$ of secondary winding and crosssection area of its conductor applying the recommendations of work [6]. Recommended $L_{\mathrm{c}}$ values make $16-22 \mu \mathrm{H}$ and $W_{1}$ is 1-2 turns. Then current amplitude value $I_{C m}$ in GVP circuit is determined by expression (8) and necessary calculation value of $A_{L}$ inductance coefficient is received by set values of $L_{\mathrm{c}}$ and $W_{1}$. Reference data given, for example in $[12,13,17,18,22]$, are used for preliminary selection of standard dimension-type of single ferrite $\Pi$ - or E-shaped core with $0.20<$ $<l_{\mathrm{c}}<0.37 \mathrm{~m}$ and $\mu_{\mathrm{e}}<1600$ and determination of effective area $A_{E C}$ of its section, as well as (using standard procedure) necessary area $A_{W}$ of core port taken by conductors of PT winding. Coefficient of turns laying, thickness of interturn and interwinding insulation and increased PT temperature at its cooling by means of natural convection are taken into account. After that, maximum possible value $H_{1}$ of magnetic field intensity in single core is calculated by formula (9) considering corresponding to them received and set values of $I_{C m}, W_{1}$ and $l_{c}$. After that, required $\mu_{\mathrm{e}}$ value for fulfillment of $B_{\text {sat }}>\mu_{0} \mu_{\mathrm{e}} H_{1}$ condition is determined by expression

$$
\mu_{\mathrm{e}} \leq \frac{B_{\mathrm{sat}}}{\mu_{0} H_{1}},
$$

where $B_{\text {sat }}$ is the allowable value of saturation induction of single ferrite core, which is recommended to be taken equal $0.35-0.40 \mathrm{~T}$. Based on this, required value of $\mu_{\mathrm{e}}$ can be provided only under condition of introduction of gap into the core. Necessary length of such gap $l_{\text {gap }}$ is determined from relationship [12]

$$
\mu_{\mathrm{e}}=\frac{\mu_{\text {init }}}{1+\mu_{\mathrm{e}} \frac{l_{\text {gap }}}{l_{\mathrm{c}}}} .
$$

Recommended values of $l_{\text {gap }}$ are from 0.5 to $1.0 \mathrm{~mm}$. If calculation results exceed recommended $l_{\text {gap }}$ values and do not provide necessary $\mu_{\mathrm{e}}$ values, than dimension-type of core with larger $l_{\text {gap }}$ should be taken, whereupon verification calculations are carried out again and final selection of dimension-type of the core is performed on their basis. In order to provide set value of $L_{\mathrm{c}}$ and required value of $A_{L}$ at taken number of turns $W_{1}$ the minimum necessary value of effective section area $A_{E}$ of the core is calculated based on received data using expression 


$$
A_{E}=\frac{l_{\mathrm{c}} A_{L}}{\mu_{0} \mu_{\mathrm{e}}}
$$

from which number $n_{\mathrm{c}}$ of single cores, making PT core, is determined as $n_{\mathrm{c}}=A_{E} / A_{E C}$ (where $A_{E C}$ is the effective section area of selected single core). At that $n$ value is rounded upwards natural number. As for the rest the calculation of PT with ferrite core is carried out using known procedures as well as recommendations given in [6].

3.2. In the case of PT with completely open rod-type ferrite core the calculation of inductance of its primary winding is started from selection of rod-type ferrite core with low initial magnetic permeability $\mu_{\text {init }}(\leq 200)$ and relationship of length of ferrite core to its diameter $l_{\mathrm{f}} / d_{\mathrm{f}}$ equal 10-14. Ferrite cores of Patron Passive Elektronic Company of $\mathrm{YC} 10 \times 100-\mathrm{YC} 10 \times 140$ or $\mathrm{YC} 13 \times 100-\mathrm{YC} 13 \times 140$ types from R2KW material [23] can be used, for example, as such a core. Then, values of effective $\mu_{\mathrm{e}}$ or relative $\mu_{\text {rel }}$ magnetic permeability of the core are determined theoretically, according to (10), or experimentally, using given in literature, in particular in [12], data and results of investigation performed earlier. After that inductance $L_{W 1}$ of PT primary winding is calculated by relationship $L_{W 1}=$ $=L_{C 1} / \mu_{\mathrm{e}}$ or $L_{W 1}=L_{C 1} / \mu_{\mathrm{rel}}$, where $L_{C 1}$ is the inductance of PT primary winding without core, determined using known procedures [12, 17, 24] for single layer coil with internal diameter equal ferrite rod diameter. Recommended values of $W 1$ turns of PT primary winding make $10-12$ and inductance $L_{W 1}$ of this winding is $6-8 \mu \mathrm{H}$ based on experience of designing, development and application of AESA. This predetermines the necessity of introduction of additional inductance $L_{\text {add }}=2-3 \mu \mathrm{H}$ in GVP circuit. PT secondary winding also should be single layer and have edgewise winding. At that internal diameter of winding is taken equal $d_{\mathrm{f}}$ and winding step $\tau_{\text {wind }}$ is such as to provide dense laying of turns of primary winding between neighboring turns of secondary winding on ferrite core. Length of winding $l_{\text {wind }}$ depending on number of turns $W 2$ of secondary winding is calculated by expression $1_{\text {wind }}=\tau_{\text {wind }}(W 2-1)$. In part number of turns $W 2$ of PT secondary winding is determined taking into account recommendations of work [6] and following from relationship

$$
W 2=W 1 \frac{U_{\text {out }}}{U_{C 0}\left(1-L_{\text {add }} / L_{W 1}\right.},
$$

where $U_{\text {out }}$ is the amplitude value of open-circuit voltage of PT secondary winding. At that considerations should be given to limitations of achievable values of $W 2$ and $U_{\text {out }}$, promoted by finite values of maximum possible length $l_{\mathrm{f}}$ of available industrially manufactured ferrite cores, that in turn, limits area of application of AESA with such PT.

Additional inductance $L_{\text {add }}$ is recommend to be performed by connecting stranded cable in form of cylindrical single-layer coil without core, outer diameter of which makes $12-20 \mathrm{~mm}$. Calculation of number of turns of such a coil by set $L_{\text {add }}$ value is carried out using known procedures, given, for example, in [12, 17].

3.3. Calculation of PT with IPC is started from selection of core material. Materials of -26 , -40 or -53 [13-17] grades are recommended by combination of electromagnetic parameters and cost indices. The main parameters of magnetization curves for these materials are given in $\mathrm{Ta}-$ ble 2 and dependencies $\mu_{\mathrm{e}}$ on $H, \mu_{\mathrm{e}} / \mu_{\text {init }}$ on $H$, and $\mu_{\mathrm{e}} / \mu_{\text {init }}$ on $B-$ in Figure 4 .

Further number of turns $W 1$ of PT primary winding, recommended values of which makes $2-4$, are set and, using recommendations of work [6] the number of turns $W 2$ of secondary winding is determined as well as type and insulation of copper strip used as conductor of this winding. Recommended width of copper strip makes 40$45 \mathrm{~mm}$, thickness is $0.2-1 \mathrm{~mm}$ and arc current density is not more than $5 \mathrm{~A} / \mathrm{mm}^{2}$. Than value of necessary area $A_{W}$ of core port is found using well-known procedures. For this total port area, taken by PT winding conductors at its cooling due to natural convection, is calculated and port height is taken equal $50-55 \mathrm{~mm}$. After that, $\mu_{\mathrm{e}}$ value is determined considering allowable values $H_{\text {max }}=4500-6000 \mathrm{~A} / \mathrm{m}$ and $B_{\max }=0.5-0.9 \mathrm{~T}$ and using diagram given in Figure 4. It usually makes 85-120 under taken assumptions depending on selected material. $I_{C m}$ value is calculated using expression (8) by setting $U_{C 0}$ from 600 to $700 \mathrm{~V}, C_{\mathrm{c}}$ from 0.5 to $4.0 \mu \mathrm{F}$ and $L_{\mathrm{c}} \geq 10 \mu \mathrm{H}$. $H_{\max }$ values is checked by expression (9) and $B_{\max }$ value by relationship $B=\mu_{0} \mu_{\mathrm{e}} H$ taking into account received values of $I_{C m}$ and $\mu_{\mathrm{e}}$, selected number of turns $W 1$ and recommended values of length of medial line of magnetic induction in core $l_{\mathrm{c}}=$ $=0.18-0.22 \mathrm{~m}$. Further, minimum necessary value of effective section area $A_{E}$ of PT core is calculated using expression (14) in form of

$$
A_{E}=\frac{L_{\mathrm{c}} l_{\mathrm{c}}}{W_{1}^{2} \mu_{0} \mu_{\mathrm{e}}} .
$$

$A_{E}$ value is used for determination of $n_{\mathrm{c}}$ constituents number of PT core of single cores applying relationship $n_{\mathrm{c}}=A_{E} / A_{E C}$ and at that rounding $n_{\mathrm{c}}$ value upwards natural number. Recommended values of effective section area $A_{E C}$ of single core are (9.4-9.8) $10^{-4} \mathrm{~m}^{2}$ and core rod size make $25 \times 38,28 \times 35,30 \times 32 \mathrm{~mm}$. PT core 
Table 2. Main parameters of IPC magnetization curves

\begin{tabular}{|c|c|c|c|}
\hline \multirow{3}{*}{ Parameter } & \multicolumn{3}{|c|}{ Value } \\
\hline & \multicolumn{3}{|c|}{ Material } \\
\hline & -26 & -40 & -52 \\
\hline $\begin{array}{l}\text { Largest allowable value of induc- } \\
\text { tance } B_{\max }, \mathrm{T}\end{array}$ & \multicolumn{2}{|c|}{1.38} & 1.40 \\
\hline Initial magnetic permeability $\mu_{\text {init }}$ & 75 & 60 & 75 \\
\hline Residual induction $B_{\mathrm{r}}, \mathrm{T}$ & 0.175 & 0.1 & 0.145 \\
\hline Coercitive force $H_{\mathrm{c}}, \mathrm{A} / \mathrm{m}$ & 40 & 37 & 510 \\
\hline \multicolumn{4}{|c|}{$\begin{array}{l}\text { Note. Saturation induction for given materials } B_{\text {sat }}=1.5 \mathrm{~T} \text { and the } \\
\text { largest allowable value of magnetic field intensity } H_{\max }= \\
=20,000 \mathrm{~A} / \mathrm{m} \text {. }\end{array}$} \\
\hline
\end{tabular}

structure and its main dimensions are determined following from $A_{W}, A_{E}$ and $A_{E C}$ values found in course of calculation taking into account its Eor $\Pi$-shaped form, after what $l_{\mathrm{c}}, H_{\max }, B_{\max }, L_{\mathrm{c}}$ and $I_{C m}$ are specified. As for the rest, similar to PT with ferrite core, known procedures as well as recommendations given in [6] are used for calculation of PT with IPC.

4. $\tau_{\text {trans }}$ duration of transition process in GVP (i.e. duration of pulses on PT windings) are determined using selected and received values $C_{\mathrm{c}}$ and $L_{\mathrm{c}}$ and expression (6) or (7), and $\tau_{\text {trans }}$ value is applied for finding necessary value of $\tau_{\text {contr }}$ duration of control pulses being fed in the control circuit of GVP switching key, which should fulfill the condition

$$
\tau_{\text {contr }} \geq 1.15 \tau_{\text {trans. }}
$$

5. Considering low frequency of passing of AESA output pulses (not more than $200 \mathrm{~Hz}$ ), their high relative pulse duration and significant levels of amplitudes of transition current and voltage in GVP circuit, domestic low-frequency thyristors of unified series T122-25 or T131-40 at $U_{C 0 \max } \leq 700 \mathrm{~V}$, and $\mathrm{T} 132-35$ or $\mathrm{T} 141-40$ at $U_{C 0 \text { max }}>700 \mathrm{~V}$, or their foreign analogs are more favorable in selection of element base of GVP switching key. Class of thyristors on repeating pulse back voltage $U_{\text {back.p }}$ are selected from the condition $U_{\text {back.p }} \geq 1.6 U_{C 0 \max }$, and maximum average $I_{\mathrm{OS} \text {.av }}$ and acting $I_{\mathrm{OS} \text {. a }}$ values of current, passing through thyristor in its open state, are determined using procedure of work [6], by expressions

$$
I_{\text {OS.av }}=0.5 I_{C m} \frac{\tau_{\text {trans }}}{T_{\mathrm{p}}}, \quad I_{\text {OS.a }}=\frac{I_{C m}}{\sqrt{\tau_{\text {trans }} / T_{\mathrm{p}}}},
$$

where $T_{\mathrm{p}}$ is the period of frequency of passing of AESA output pulses (turn-on frequency of GVP switching key).

6. Back diode of key (see Figure 1,c) in designing GVP switching key with two-way conductance is selected from the condition
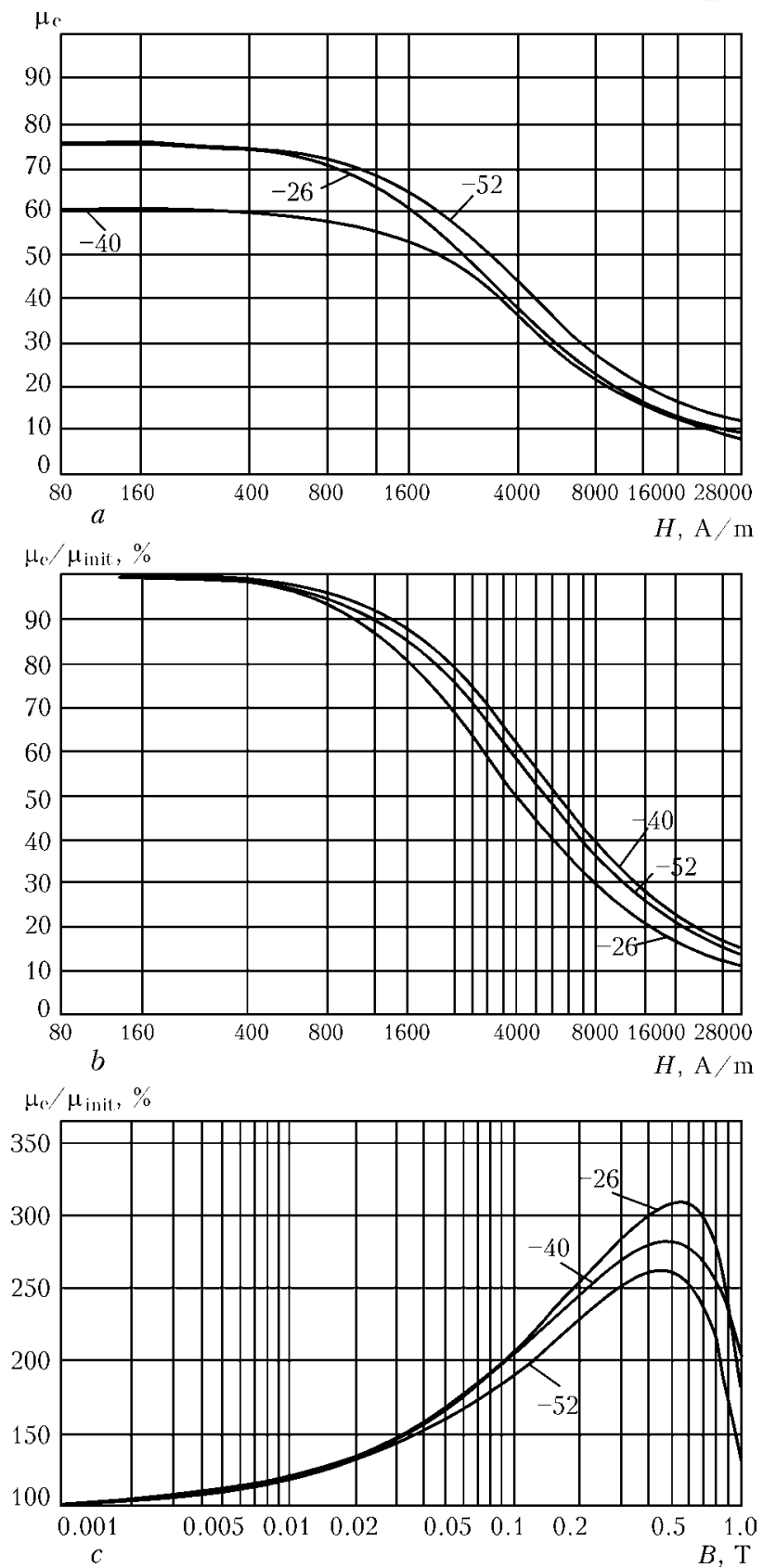

Figure 4. Diagrams of $\mu_{\mathrm{e}}(a)$ and $\mu_{\text {init }}(b)$ dependence on $H$, and $\mu_{\mathrm{e}} / \mu_{\text {init }}$ dependence on $B(c)$ in PT with IPC

$U_{R} \geq 1.6 U_{C 0 \max }, \quad I_{F(A V)} \geq 0.8 I_{\text {OS.av }}, \quad I_{F S M} \geq I_{C m}$,

where $U_{R}$ is the maximum allowable back voltage of diode; $I_{F(A V)}$ is the maximum allowable value of direct current passing through diode; $I_{F S M}$ is the maximum allowable value of repeating direct pulse current passing through diode. High-voltage fast diodes with small values of the largest forward drop $\left(U_{F} \leq 1.5 \mathrm{~V}\right)$ and reverse recovery time $\left(t_{r r} \leq 150 \mathrm{~ns}\right)$ are the most favorable. For example, fast rectifying diode VS-40EPF1 of Vishay Semiconductor company [25] meets these requirements.

Peculiarities of analysis and calculation of AESA with CD built based on application of 
resonant pumping method. CD built on application of method of resonant pumping are widely used in medical equipment, measuring and radar engineering, in laser devices, in electrotechnological units, devices of automobile electronics and many other technical fields. Such CDs have found application in boosters of units for arc and plasma welding $[1,26]$. Principle of operation of such CD is based on the fact that GVP capacitive storage is the element of resonant $L C$-circuit, including series current-limiting charge choke, pickoff diode and capacitor of GVP forming circuit being recharged at each turn-on of switching key of this circuit. At that, charge voltage $U_{C 0}$ of GVP capacitive storage in steady-state mode can significantly exceed CD input voltage. In theory, process of increase of $U_{C 0}$ value can go into infinity, however, practically achievable value of voltage of $U_{C 0}$ charge exceeds value of $C D$ input voltage not more than in 3-5 times [26] due to finite values of Qfactor in real resonant circuits of charge and discharge of GVP capacitive storage and inevitable ohmic losses in their elements.

Basic variant of AESA with CD scheme, built based on application of method of resonant pumping, are given in Figure 5, $a$. Such AESA (as AESA with CD based on key scheme with dosing reactor) is characterized by wide range of possible levels of input voltage $U_{\text {in }}$ (in most cases making from 100 to $350 \mathrm{~V}$ ) as well as invariance of such CD by nature of AESA supply voltage. At that, normal functioning of CD in AESA supply with alternating current voltage does not depend on its frequency. If only direct current voltage is supposed for AESA supply, than presence in scheme, given in Figure 5, $a$, of rectifier $U Z 1$ and buffer capacitor $C 1$ is not strictly necessary.

Charging of capacitive storage (capacitor $C 2$ ) of GVP forming circuit in AESA with CD (see Figure $5, a$ ) is carried out by special mode called resonant. Feeding of supply voltage $U_{\text {sup }}$ to AESA promotes gradual buildup by sine law of charge current $i_{C}$ of capacitive storage (capacitor $C 2$ ), and $u_{C}$ charge voltage of this capacitor by exponential law. At the time, when $u_{C}=U_{\text {in }}$ is provided, charge current $i_{C}$ will achieve its amplitude value $I_{m}$, after what $i_{C}$ current starts drop also by sine law, that promotes emf of self-induction on $L 1$ choke, acting in accordance with $U_{\text {in. }}$. As a result of this, charging of capacitor $C 2$ will continue up to the moment when voltage $u_{C}$ on it reaches virtually double value $U_{\text {in }}$, and, because of presence of $V D 1$ diode, such a level of $u_{C}$ voltage of $C 2$ capacitor charge will remain up to the moment of turn-on of GVP switching key (thyristor VS1). Turn-on of thyristor $V S 1$ provides for beginning of process of capacitor $C 2$ re-charging in GVP forming circuit via PT primary winding (transformer $T V 1$ ). Duration of process of $C 2$ capacitor re-charging is determined by semi-period of own oscillations of GVP forming circuit and does not depend on frequency of turn-on of its switching key (thyristor VS1). Recharging current (current in GVP circuit) will reach its maximum value $I_{C m}$ in the moment when key voltage on it reduces to zero in process of capacitor $C 2$ re-charging. After that, re-charging current drop by sine law will start, that promotes appearance of emf of self-induction on inductance of PT primary winding (transformer $T V 1$ ), providing passing of current in GVP circuit in the interval from $\pi / 2$ to $\pi$ of semi-period of own oscillations. Voltage of capacitor $C 2$ at the end of re-charging process will be close in modulus to value of voltage before re-charging beginning and negative to the latter by polarity. At that re-charging current drops virtually to zero, due to what thyritor VS1 will turn-off. From this moment second cycle of charge of capacitive storage (capacitor C2) of GVP forming circuit is started, however, in this case voltage and charge current will be determined by sum of voltage $U_{\text {in }}$ and acting in accordance with it steady-state recharging voltage of capacitor $C 2$. This will promote increase of voltage on $C 2$ capacitor up to the value close, at least, to four-fold value of $U_{\text {in }}$ to the moment of ending of charging cycle.

Further all processes in CD, scheme of which is given in Figure 5, $a$, are repeated similar to described above. Voltage in the third cycle of charge can only insignificantly exceed charge voltage in the second cycle due to finite values of Q-factor of charge and discharge circuits of GVP capacitive storage. It was experimentally stated that charge voltage of GVP capacitive storage (capacitor C2) can be considered as steady-state one starting from the moment of ending of third charge cycle.

Equivalent circuit of CD, built based on application of resonant pumping mode, is given in Figure 5, b. In this circuit $R=R_{L 1}+R_{\mathrm{CLR}}+$ $+E S R_{C 1}+E S R_{C 2}+r_{\mathrm{dyn}}+R_{I}$ is the active resistance of charge circuit. Here, $R_{L 1}$ is the $L 1$ choke active resistance; $R_{\mathrm{CLR}}$ is the active resistance of current-limiting resistor $R 1 ; E S R_{C 1}$ and $E S R_{C 2}$ are the equivalent active series resistances of $C 1$ and $C 2$ capacitors, respectively; $r_{\text {dyn }}$ is the dynamic resistance of $V D 1$ diode; $R_{I}$ is the active resistance of $T V 1$ transformer primary winding); $L=L_{\text {choke }}+L_{I}\left(L_{\text {choke }}-\right.$ inductance of choke $L 1 ; L_{I}$ is the inductance of $T V 1$ transformer primary winding); $C$ is the capacity of GVP capacitive storage (capacitor $C 2$ ). Since $R_{C L R}>R_{L 1}+$ 
$+E S R_{C 1}+E S R_{C 2}+r_{\text {dyn }}+R_{I}$, and $L_{\text {choke }}>L_{I}$, than $R=R_{\mathrm{CLR}}$ and $L=L_{\text {choke }}$ can be assumed with sufficient level of accuracy.

It can be seen from CD equivalent circuit (see Figure $5, b$ ) that charge circuit of GVP capacitive storage (capacitor $C 2$ ) represents itself series $L C R$-circuit. Process of charging of capacitive storage of such circuit for $i_{C}$ current and $u_{C}$ voltage is described by known differential equations $[8,9]$. At that, variant with zero initial condition (i.e. in the moment of cycle beginning $i_{C}=0$, $\left.u_{C}=0\right)$ is considered for the first charge cycle, and that for the second and further cycles is the variant with non-zero initial conditions, at which at the moment of beginning of each cycle $i_{C}=0$ and $u_{C}=-U_{C 0}$, where $U_{C 0}$ is the steady-state charge voltage of GVP capacitive storage. According to $[8,9]$ and considering peculiarities of circuits, given in Figure 5, solutions of these equations for the first cycle of charge of GVP capacitive storage can be represented in form of

$$
\begin{gathered}
i_{C}=\frac{U_{\text {in }}}{\omega^{\prime} L} e^{-\delta T} \sin \omega^{\prime} t ; \\
u_{C}=U_{\text {in }}+U_{\text {in }} \frac{\omega_{0}}{\omega^{\prime}} e^{-\delta t} \cos \left(\omega^{\prime} t-\gamma\right),
\end{gathered}
$$

where $\delta=R / 2 L ; \omega_{0}=1 \sqrt{L C} ; \omega^{\prime}=\sqrt{\omega_{0}^{2}-\delta^{2}}$; $\operatorname{tg} \gamma=\delta / \omega^{\prime}$, and for the second and further charge cycles in form of

$$
\begin{gathered}
i_{C}=-\frac{U_{\text {in }}+(N-1) U_{C 0}}{\omega^{\prime} L} e^{-\delta t} \sin \omega t ; \\
u_{C}=\left(U_{\text {in }}+U_{C 0}\right) e^{-\delta t} \cos \left(\omega^{\prime} t-\gamma\right),
\end{gathered}
$$

where $N=-U_{C 0} / U_{\text {in }}$ is the coefficient of voltage increment on GVP capacitive storage to the level preceding beginning of charge cycle. As shown in [27], charge voltage $U_{C 0}$ of GVP capacitive storage (capacitor $C 2$ ) in steady-state mode can be determined by formula

$$
U_{C 0}=U_{\text {in }} \text { cth }\left(\frac{\pi}{2 \sqrt{\frac{4 L}{R^{2} C}-1}}\right),
$$

and amplitude value $I_{m}$ of charge current $i_{C}$ by expression

$$
I_{m}=U_{\text {in }}\left(1+\operatorname{cth} \frac{\pi}{2 \gamma}\right) \sqrt{\frac{C}{L}} e^{\frac{-1}{\gamma} \operatorname{arctg} \gamma},
$$

where $\gamma=\sqrt{\frac{4 L}{R^{2} C}-1}$.

Relationships (19) and (20) are true under condition $C 1>C 2$, that is virtually always fulfilled in AESA with CD built based on resonant pumping method. It also follows from expressions (19) and (20) that resistor $R 1$ is not only cur-

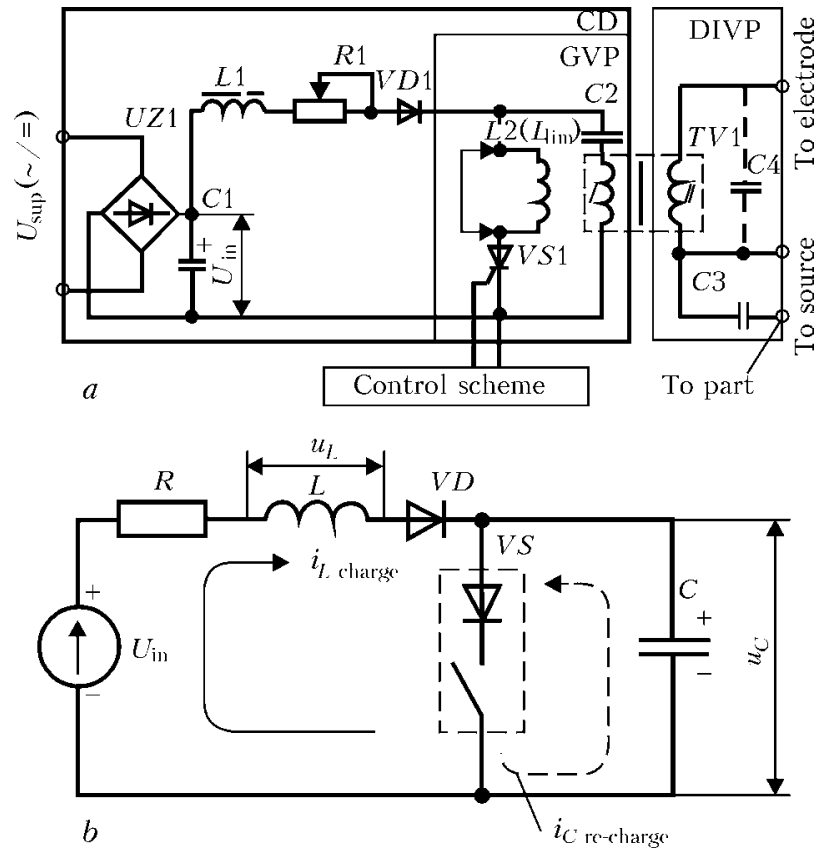

Figure 5. Electric schematic diagram of basic variant of AESA with CD based on application of method of resonant pumping $(a)$, and scheme of equivalent circuits of charge and re-charge of GVP capacitive storage of basic variant of AESA with CD based on application of method of resonant pumping (b)

rent-limiting element, but element allowing limiting steady-state charge voltage of GVP capacitive storage at set level. Duration $\tau_{\text {charge }}$ of pulses of charge current $i_{C}$ is determined by semi-period of frequency $\omega^{\prime}$ by formula

$$
\tau_{\text {charge }}=\frac{\pi}{\sqrt{\frac{1}{L C}-\frac{R^{2}}{4 L^{2}}} .}
$$

Diagrams of $i_{C}$ current, $u_{C}$ and $u_{L}$ voltages, representing nature of transition processes in CD and GVP in AESA, built using resistant pumping method, are given in Figure 6.

Calculation of parameters and selection of components of charge circuit of CD built based on application of resonant pumping method can be performed in the following way.

1. Calculation and nominal values of GVP capacitive storage and type of applied capacitors are determined based on recommendations given in $[6,7]$ and in present work. At that, it is proceeded from the fact that steady-state charge voltage $U_{C 0}$ of GVP capacitive storage makes $550 \pm 50 \mathrm{~V}$ in the majority of cases.

2. Expression (22) is used for determination of amplitude value $I_{m}$ of charge current $i_{C}$. Recommended $I_{m}$ values make 0.9 to $1.5 \mathrm{~A}$ according to results of carried investigations and experimental data, depending on set values of $C, U_{\text {in }}$ or $U_{C 0}$, at that lower $C, U_{\text {in }}$ or $U_{C 0}$ values correspond to lower $I_{m}$ values. 


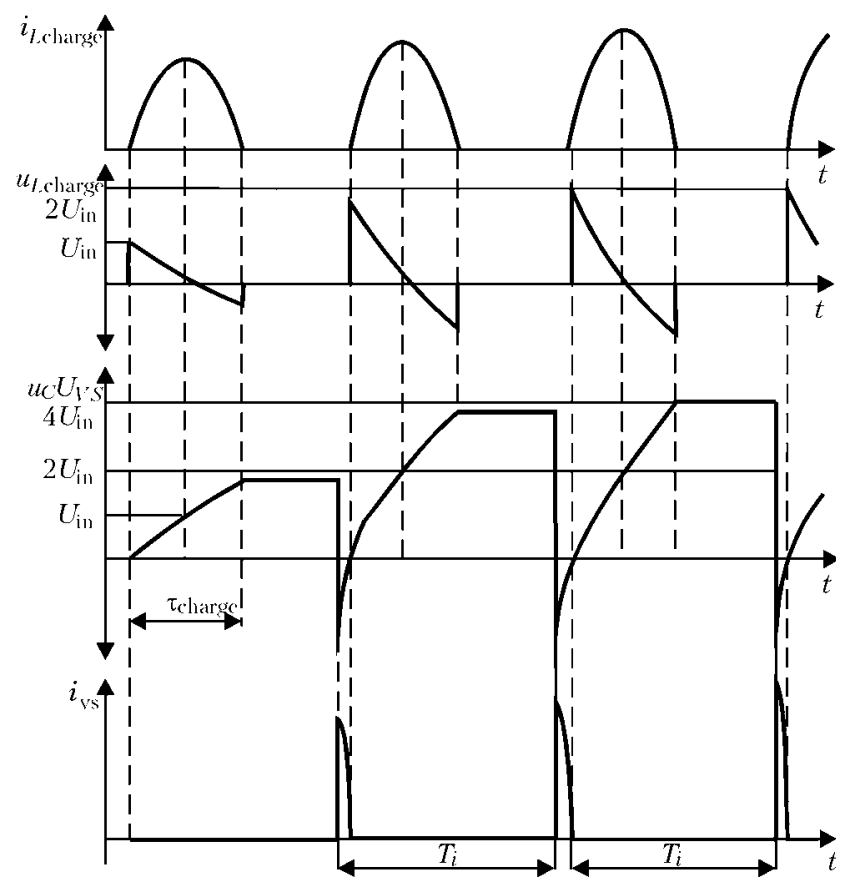

Figure 6. Diagrams of currents and voltages in CD circuits and GVP of AESA with CD based on application of method of resonant pumping

3. Assuming that inductance of charging choke $L 1$ (see Figure 5, $a$ ) is linear and does not depend on current $i_{C}$ passing though choke, and using calculated or selected values of $I_{m}$ and $C$ as well as set values of $U_{\text {in }}$ and energy $W_{C}$ accumulated in GVP capacitive storage, $L$ value is determined by expression

$$
L \geq \frac{C U_{C 0}^{2}}{I_{m}} .
$$

Recommended values of inductance of charging choke $L 1$ make 65 to $200 \mathrm{mH}$, coming from range of $I_{m}$ recommended values, expression (24) and experience of designing of AESA with CD based on resonant pumping method. The most reasonable choke design is a shell-type structure. It can be realized based on cores from standard E-shaped plates or strip magnetic conductors from thin coldrolled anisotropic electrical steel of 3411-3414 grades. At that, length of total diamagnetic gap in the core of charging choke should make 0.9$1.5 \mathrm{~mm}$. Electromagnetic and design calculations of this choke can be carried out by known procedures, given, for example, in [12,17].

4. The following things should be considered in determination of resistance $R$. Namely, first of all, $R \approx R 1$ (see Figure $5, a$ ), and secondly that application of initial (virtually linear) section of exponent is desirable to be used in charging of GVP capacitive storage, and $L / R \leq \tau_{\text {charge }}$ condition should be fulfilled for this. Taking into account results of calculations and experimental data, the recommended values of resistor resis- tance $R 1$ make from 47 to $120 \mathrm{Ohm}$, moreover the more $U_{\text {in }}$ value is, the more these values are. The largest dissipation value $P_{R 1 \text { max }}$, emitted in resistor $R 1$, can be determined by known expression $P_{R 1 \max }=I_{\mathrm{act}} R 1$, where $I_{\mathrm{act}}=$ $0.707 I_{m} / \sqrt{T / \tau_{\text {charge }}}$ is the maximum acting current value $i_{C}$ passing via resistor $R 1 ; T$ is the period of frequency of pulses of charge current (frequencies $f_{\mathrm{p}}$ of turn-on of GVP switching key). $P_{R 1 \max }=26.66 \mathrm{~W}$ at $I_{m}=1.5 \mathrm{~A}, R 1=$ $=120 \mathrm{Ohm}, \tau_{\text {charge }}=1 \mathrm{~ms}$ and $f_{\mathrm{p}}=200 \mathrm{~Hz}$. At the same values of $I_{m}, R 1, \tau_{\text {charge }}$ and $f_{\mathrm{p}}=$ $=100 \mathrm{~Hz}, P_{R 1 \max }=13.44 \mathrm{~W}$, and $P_{R 1 \max }=$ $=6.92 \mathrm{~W}$ at $f_{\mathrm{i}}=50 \mathrm{~Hz}$. It follows from this that recommended area of frequencies $f_{\mathrm{p}}$ is limited by values from 80 to $120 \mathrm{~Hz}$, and in the case of AESA application only for initial excitation of direct current arc the nominal power of resistor $R 1$ can be significantly reduced in comparison with calculation one and make 4-6 W. Power resistors of $\mathrm{C} 5-\mathrm{C} 35$ or SPS, SQZ, SQHG series [28] are reasonable to be used as $R 1$ resistor.

5. The main parameters for selection of $V D 1$ diode (see Figure 5, a) are maximum allowable value of average direct current $I_{F}$ and back voltage $U_{R}$ as well as maximum values of direct drop of voltage $U_{F}$ and reverse recovery time $t_{r r}$. At that diode parameters should fulfill the conditions

$$
U_{R \max } \geq 1.2 N U_{\mathrm{in}}, \quad I_{F \max } \geq 1.2 I_{\mathrm{av} . \max }
$$

where $I_{\mathrm{av} \text { max }}$ is the maximum average value of charge current of GVP capacitive storage, which can be determined using expression

$$
I_{\text {av. max }}=0.637 I_{m} \tau_{\text {charge }} / T .
$$

Calculation of parameters and selection of GVP components of AESA, built based on application of resonant pumping method, do not differ from given in present work for basic variant of GVP with switching key of unidirectional conduction.

If AESA (including one containing CD, built based on resonant pumping) is designed not only for initial excitation, but for stabilizing of process of burning of arc with low stability, than efficiency of AESA work at unidirectional conduction of GVP switching key can be reached with the help of additional high-frequency circuit of impact excitation, formed by inductance of PT secondary winding and parallel to this winding capacity of high voltage capacitor (for example, KVI-3 series), designated as $C_{\mathrm{C} 2}$ in Figure 1, $a$, or $C 4$ in Figure 5, $a$. Recommended values of nominal capacity of such capacitor is from 1000 to $3300 \mathrm{pF}$.

It should be noted that currently AESA with $\mathrm{CD}$ based on application of resonant pumping method find more and more distribution and is 
used as independent lowering units (for example, exciter-stabilizers VSD-01 U3) [1] for equipping arc welding stations as well as for functional blocks, imbedded in welding equipment, mainly in welding power supply sources (for example, power supply ITs 616 U13.1 and ITs 617 U3.1 for TIG welding) [29].

\section{Conclusions}

1. GVP design with step-up PT with completely open rod-type ferrite core is the most reasonable for AESA, pulse energy of which is not more than $0.1 \mathrm{~J}$ and which are designed only for excitation of arc in TIG welding. AESA, energy of generated pulses of which makes from 0.10 to $0.25 \mathrm{~J}$, can apply PT with ferrite core of $\Pi$ - or E-shaped form with total gap to $1 \mathrm{~mm}$. Using of iron powder cores in PT is reasonable virtually in all possible range of energies of AESA generated pulses, including at $1 \mathrm{~J}$ level and more.

2. AESA with CD, based on application of method of resonant pumping, the same as $\mathrm{CD}$, based on key scheme with dosing reactor, do not depend on nature of AESA supply voltage and capable to function in supply with direct as well as alternating current of harmonic or rectangular or close to them forms of frequency from tens of hertz (including $50 \mathrm{~Hz}$ ) to tens of kilohertz. The first and the second AESA virtually have no limitations on technologically grounded levels of energy of generated pulses, which can make from 0.01 to $1 \mathrm{~J}$ and more. Both AESA have wide area of application in arc and plasma welding and can be used in traditional power supplies as well as in invertor-type power supplies. Such AESA can be efficiently applied in manual methods of arc and plasma welding and in automatic welding machines, in particular, in single- and multi-station systems for automatic orbital TIG welding of pipeline joints. The advantage of AESA with CD, based on application of resonant pumping method, is the fact that only one controlled semi-conductor key and significantly simpler control scheme are necessary for its construction. The advantage of AESA, based on key scheme with dosing reactor, is wider range of possible values of supply voltage.

3 . Engineering procedure for calculation of basic variant of GVP as well as CD, based on application of resonant pumping method, is developed as a result of analysis considering experience of development, designing, manufacture and operation of AESA. Recommendations on selection of their element base are worked out that can be useful not only for AESA development, but for other similar devices designed for application in different branches of science and technology.
1. (1986) Equipment for arc welding: Refer. Book. Ed. by V.V. Smirnov. Leningrad: Energoatomizdat.

2. Temkin, B.Ya. (1981) Theory and calculation of welding arc exciters: Syn. of Thesis for Cand. of Techn. Sci. Degree. Leningrad.

3. Dudko, D.A., Fedotenkov, V.G., Makhlin, N.M. (1980) Thyristor pulse generators of UPD-1 type. Avtomatich. Svarka, 6, 61-63.

4. Paton, B.E., Zaruba, I.I., Dymenko, V.V. et al. (2007) Welding power sources with pulse stabilizing of arcing. Kyiv: Ekotekhnologiva.

5. Fedotenkov, V.G., Makhlin, N.M., Temkin, B.Ya. (1981) Pulse generator for excitation and stabilizing of alternatiing current arc. Svarochn. Proizvodstvo, 8, 33-34.

6. Makhlin, N.M., Korotynsky, A.E. (2014) Analysis and procedure of calculation of series connection electronic devices for contactless arc excitation. The $\mathrm{Pa}$ ton Welding J., 1, 30-40.

7. Makhlin, N.M., Korotynsky, A.E. (2015) Asynchronous exciters and stabilizers of welding arc. Analysis and design procedure. Pt 1. Ibid., 3/4, 24-35.

8. Atabekov, G.I. (2009) Theoretical principles of electrical engineering. Linear electric circuits: Manual. 7 th ed. St.-Petersburg: Lan.

9. (2008) Theoretical principles of electrical engineering: Handbook on theory of electrical circuits. Ed. by Yu.A. Bychkov et al. St.-Petersburg: Piter.

10. Leskov, G.I. (1970) Electric welding arc. Moscow: Mashinostroenie.

11. Zamyatin, V.Ya., Kondratiev, B.V., Petukhov, V.M. (1988) Power semi-conductor devices. Thyristors: Handbook. Moscow: Radio i Svyaz.

12. Baev, E.F., Fomenko, L.A., Tsymbalyuk, V.S. (1976) Inductive elements with ferromagnetic cores. Moscow: Sov. Radio.

13. http://www.coretech.com.uahttp://www.microme tals.com

14. Cox, J. (2006) Iron power cores in pulse power sources. Pt 1. Sovrem. Elektronika, 1, 58-65.

15. Cox, J. (2006) Iron power cores in pulse power sources. Pt 2. Ibid., 2, 66-69.

16. Cox, J. (2006) Iron power cores in pulse power sources. Pt 3. Ibid., 3, 70-75.

17. Kalantarov, P.L., Tsejtlin, L.A. (1986) Calculation of inductances: Refer. Book. Leningrad: Energoatomizdat.

18. http: / / www.epcos.com

19. http://www.icel.it

20. Handbook on electrotechnical materials. Ed. by Yu.V. Koritsky et al. 3rd ed. Leningrad: Energoatomizdat.

21. Belorussov, N.I., Saakyan, A.E., Yakovleva, A.I. (1987) Electric cables, wires, cords: Handbook. Ed. by N.I. Belorussov. 5th ed. Moscow: Energoatomizdat.

22. Sidorov, I.N., Khristinin, A.A., Skornyakov, S.V. (1989) Small-sized magnetic conductors and cores: Handbook. Moscow: Radio i Svyaz.

23. http: / / www.patron-components.com

24. Volgov, V.A. (1977) Parts and units of electronic devices. Moscow: Energiya.

25. http://www.vishay.com

26. Belinsky, S.M., Kagansky, B.A., Temkin, B.Ya. (1975) Equipment for nonconsumable-electrode inertgas welding. Leningrad: Energiya.

27. Pentegov, I.V. (1982) Bases of theory of charging circuits of capacitor storage. Kiev: Naukova Dumka.

28. http://www.dart.ru/cataloguenewresistors / ty / h tml/cement.shtml

29. Makhlin, N.M., Korotynsky, A.E., Bogdanovsky, V.A. et al. (2011) Single- and multioperator systems for automatic welding of position butt joints of nuclear power plant piping. The Paton Welding J., 11, 28-36. 